\title{
New heterotic non-Kähler
}

\section{geometries}

\section{Melanie Becker ${ }^{1}$, Li-Sheng Tseng ${ }^{2,3}$ and Shing-Tung Yau $^{2}$}

${ }^{1}$ George P. and Cynthia W. Mitchell Institute for Fundamental Physics, Texas A \& M University, College Station, TX 77843, USA mbecker@physics.tamu.edu

${ }^{2}$ Department of Mathematics, Harvard University, Cambridge, MA 02138, USA

tseng@math.harvard.edu; yau@math. harvard.edu

${ }^{3}$ Center for the Fundamental Laws of Nature, Jefferson Physical Laboratory, Harvard University,

Cambridge, MA 02138, USA

\begin{abstract}
New heterotic torsional geometries are constructed as orbifolds of $T^{2}$ bundles over $K 3$. The discrete symmetries considered can be freely acting or have fixed points and/or fixed curves. We give explicit constructions when the base $K 3$ is Kummer or algebraic. The orbifold geometries can preserve $\mathcal{N}=1,2$ supersymmetry in four dimensions or be non-supersymmetric.
\end{abstract}

\section{Introduction}

Heterotic string compactifications play a prominent role in string theory model building as the standard model gauge group can be easily incorporated

e-print archive: http://lanl.arXiv.org/abs/arXiv:0807.0827 
in this approach. Some recent progress in heterotic model building was made in [1], where a heterotic M-theory model was constructed that reproduces the charged particle content of the MSSM without additional exotics. ${ }^{1}$ Interestingly, F-theory models that incorporate GUT gauge groups have also recently been constructed in the literature $[5,6]$. Common to these recent developments is that moduli fields coming either from metric deformations and/or deformations describing brane positions are not stabilized, so that making predictions for physical observables become challenging.

Now more than ever it is important to develop techniques to construct heterotic flux compactifications in which moduli stabilization can be addressed. Having a heterotic three generation model with stabilized moduli allows us to remove this (uncharged) "exotic" matter and in principle predict the masses of quarks and leptons, an important step towards connecting string theory to the real world.

Heterotic flux compactifications have been known for quite some time, starting with the seminal works of [7-9] in the mid-1980s. All the more, it is surprising that the number of torsional geometries that can serve as backgrounds for heterotic string compactifications seems rather limited. The most studied class of smooth heterotic torsional compactifications is the FSY geometry $[10,11]$. The manifold is a $T^{2}$ bundle over a $K 3$ surface $[12,13]$ similar to the well-known non-Kähler torus bundle construction of Calabi and Eckman [14]. The solution was first identified as the heterotic dual of M-theory on $K 3 \times K 3$ with non-zero $G$-flux $[15,16]$. It was proven in [10] to satisfy both the requirements of supersymmetry and the anomaly condition of the heterotic theory. A conformal field theory description in the language of a gauged linear sigma model has also been developed in [17].

As was discussed in $[13,18]$, this model has a vanishing Euler characteristic as well as a vanishing number of generations. When the spin connection is embedded into the gauge connection, the net number of generations is given by the Euler characteristic of the internal Calabi-Yau geometry [19]. However, for the more general heterotic flux compactifications that we are interested in, where the spin connection cannot be embedded into the gauge connection, the net number of generations is given by one-half of the third Chern number of the gauge bundle [18].

Our goal here is to expand the set of consistent heterotic torsional geometries by constructing orbifolds of the FSY geometry. Orbifolding techniques [20] were already used in the early days of string model building

\footnotetext{
${ }^{1}$ Other standard model motivated heterotic constructions are given in $[2,3]$. A CFT model with no exotics has also been presented in [4].
} 
to construct models with a small number of generations. Modding out some heterotic string compactifications with an unrealistically large number of generations by a discrete symmetry, led to more realistic models (see, e.g., $[21,22])$. In fact, the first heterotic three generation model was constructed by quotienting a complete intersection Calabi-Yau three-fold [21].

To construct orbifolds of a $T^{2}$ bundle over $K 3$ surface, we use special classes of $K 3$ base surfaces. One set of models arises from Kummer $K 3$ surfaces which have a $T^{4} / \mathbb{Z}_{2}$ orbifold limit. A second set of models uses algebraic $K 3$ surfaces with finite discrete group actions. The simplest ones are those with a branched covering description. The orbifold actions described in this approach are either freely acting or have fixed curves and/or fixed points. The freely acting orbifold actions give smooth geometries which may preserve $\mathcal{N}=0,1,2$ supersymmetry in four dimensions. For the non-freely acting discrete symmetries, the resulting orbifolds will contain singularities which geometrically need to be resolved. Physically, if the resolution can involve turning on non-vanishing gauge bundles, this may lead to models with a non-zero third Chern class. Unfortunately, unlike the CalabiYau case where orbifold singularities can be straightforwardly resolved by means of toric resolution (see reviews in [23-25]), singularity resolution in the non-Kähler context seems much more involved and has not been studied previously. The required analysis is delicate and rather technical, and hence, we will leave the discussion of non-Kähler resolution for a subsequent work.

The organization of this paper is as follows. In Section 2, we review the properties of torsional manifolds that we need in the later sections and describe the FSY geometry in some detail. In Section 3, we discuss properties of the orbifolds of the FSY geometry, i.e., $Y=X / \Gamma$ where $\Gamma$ is a discrete symmetry group of $X$, a torus bundle over $K 3$ geometry. The discrete symmetries can act on the torus fiber either as a shift or a rotation. In the former case the orbifold actions are always freely acting and lead to smooth models with vanishing Euler characteristic and third Chern class. In the latter, the orbifold action may have fixed points and fixed curves singularities. In Section 4, we construct models that realize these different possibilities starting from the FSY geometry with the $K 3$ base in the $\mathbb{Z}_{2}$ orbifold limit $T^{4} / \mathbb{Z}_{2}$. In Section 5 , we take as the base $K 3$ those with a branched covering description to build a second set of orbifold torsional geometries. Section 6 discusses constructions based on more general algebraic $K 3$ base with a discrete symmetry group action. In particular, we give a supersymmetric orbifold model with only fixed point singularities. Orbifold models with only fixed points should be easier to resolve than those containing fixed curves. In Section 7, we present our conclusions and raise some open questions. 


\section{Review of torsional geometry}

The internal six-dimensional complex geometries of supersymmetric flux compactifications of the heterotic string are characterized by a hermitian $(1,1)$-form $J$, a holomorphic (3,0)-form $\Omega$, and a non-abelian gauge field strength $F$ that are constrained by supersymmetry and anomaly cancellation conditions [7]

$$
\begin{aligned}
& d\left(\|\Omega\|_{J} J \wedge J\right)=0, \\
& F^{(2,0)}=F^{(0,2)}=0, \quad F_{m n} J^{m n}=0, \\
& 2 \mathrm{i} \partial \bar{\partial} J=\frac{\alpha^{\prime}}{4}[\operatorname{tr}(R \wedge R)-\operatorname{tr}(F \wedge F)] .
\end{aligned}
$$

These equations (sometimes termed the "Strominger system" in the mathematics literature) provide the necessary and sufficient conditions for spacetime supersymmetry in four dimensions and imply the equations of motion to one-loop order in $\alpha^{\prime}$. Given a solution, the physical fields $\left(g, H_{3}, \phi\right)$ are expressed in terms of $(J, \Omega)$ as

$$
\begin{aligned}
g_{a \bar{b}} & =-\mathrm{i} J_{a \bar{b}}, \\
H_{a b \bar{c}} & =-\mathrm{i}\left(\partial_{a} J_{b \bar{c}}-\partial_{b} J_{a \bar{c}}\right), \\
\mathrm{e}^{-4 \phi} & =\frac{\mathrm{i}}{3 !} \Omega_{a b c} \bar{\Omega}_{\bar{a} \bar{b} \bar{c}} J^{a \bar{a}} J^{b \bar{b}} J^{c \bar{c}},
\end{aligned}
$$

where we have set the integrable complex structure to take the diagonal form $J_{a}^{b}=\mathrm{i} \delta_{a}^{b}$.

The most well-known solution to these equations consists of the complex $T^{2}$ bundle over a $K 3$ surface which we denote by $X{ }^{2}{ }^{2}$ The explicit form of the holomorphic $(3,0)$-form for this manifold is

$$
\Omega=\Omega_{K 3} \wedge \theta,
$$

where $\Omega_{K 3}$ is the holomorphic $(2,0)$-form on the $K 3$ base and $\theta=(d z+\alpha)$ (with $z$ the fiber coordinate and $\alpha$ a connection one form on $K 3^{3}$ ) is defined to be a global $(1,0)$-form. The hermitian $(1,1)$-form $J$ comes from the

\footnotetext{
${ }^{2} \mathrm{~A} T^{4}$ base geometry can satisfy the necessary $\mathrm{SU}(3)$ intrinsic torsion conditions of conformally balanced (2.1) and the existence of a holomorphic three form [26]. It would however not be consistent with the anomaly condition [11,27].

${ }^{3}$ To be rigorous, $\alpha$ should be the pull-back of the connection one form on $K 3$ to $X$.
} 
Kähler form of the $K 3$ and the metric on the torus bundle

$$
J=\mathrm{e}^{2 \phi} J_{K 3}+\frac{\mathrm{i}}{2} \frac{A}{\tau_{2}}(d z+\alpha) \wedge(d \bar{z}+\bar{\alpha}),
$$

where the moduli of the torus are parametrized by the area $A$ and the complex structure $\tau=\tau_{1}+\mathrm{i} \tau_{2}$. The dilaton $\phi$ is non-constant and depends on the base coordinates only. Supersymmetry demands that the curvature of the torus bundle is of type $(2,0) \oplus(1,1)$

$$
\omega=\omega_{1}+\tau \omega_{2}=d \theta=d \alpha \in H^{(2,0)} \oplus H^{(1,1)}(K 3) \quad \text { with } \omega_{1}, \omega_{2} \in H^{2}(K 3, \mathbb{Z}) .
$$

Additionally, $\omega$ is required to be primitive with respect to the base

$$
\omega \wedge J_{K 3}=0
$$

Turning on a $(2,0)$ component for $\omega$ reduces the $\mathcal{N}=2$ four-dimensional supersymmetry to $\mathcal{N}=1$, whereas a $(0,2)$ component breaks supersymmetry completely. ${ }^{4}$

The solution also includes a gauge bundle with a gauge field strength that satisfies the hermitian-Yang-Mills conditions (2.2). The field strength is related to the metric and curvature two form by the anomaly equation (2.3). Integrating the anomaly equation over the base $K 3$ leads to the topological condition

$$
-\frac{1}{16 \pi^{2}} \int_{K 3} \operatorname{tr} F \wedge F-\frac{A}{\tau_{2}} \int_{K 3} \omega \wedge \bar{\omega}=24
$$

where $\bar{\omega}=\omega_{1}+\bar{\tau} \omega_{2}$ is the complex conjugate of $\omega$. This is the main sufficiency condition to ensure that the anomaly equation, which for these geometries, can be interpreted as a non-linear second-order partial differential equation for $\phi$, can be solved $[10,11]$.

As for ordinary Calabi-Yau manifolds (see [19]), it was shown in [18] that the net number of generations is determined by an index which (for bundles with $c_{1}(F)=0$, such as the $S U(N)$ bundles we are considering) is related to the third Chern number of the bundle

$$
N_{\text {gen }}=\frac{1}{2} C_{3}(F) .
$$

\footnotetext{
${ }^{4} \mathrm{~A}$ derivation of these supersymmetry constraints can be found in the appendix of [28].
} 
More concretely, the third Chern class of the bundle is

$$
c_{3}(F)=-\frac{\mathrm{i}}{48 \pi^{3}}[2 \operatorname{tr}(F \wedge F \wedge F)-3 \operatorname{tr}(F \wedge F) \wedge \operatorname{tr} F+\operatorname{tr} F \wedge \operatorname{tr} F \wedge \operatorname{tr} F],
$$

where the trace is taken over the generators of the Lie-algebra. The generators are traceless for $\mathrm{SU}(N)$ gauge groups, so that trivially the first Chern class, $c_{1}(F)=0$. Thus for $\mathrm{SU}(N)$ bundles, the last two contributions to the above formula vanish and integrating the first term gives the index of the Dirac operator $[29,30]$. If the spin connection is embedded into the gauge connection (which is not the case of interest here) this formula reduces to the more familiar expression $N_{\text {gen }}=\chi / 2$, where $\chi$ is the Euler characteristic of the internal manifold. It was shown in [11] that all stable gauge bundles on $X$ that can satisfy the anomaly condition (2.3) are those obtained by lifting stable bundles on $K 3$ to $X$. From (2.13), it then becomes evident that the net number of generations vanishes. Moreover, the existence of a non-vanishing vector field along the torus fiber implies that the Euler characteristic $\chi(X)=0[12,13]$.

Some of the new torsional manifolds we construct in the next sections will have a non-vanishing Euler characteristic. Although not given here, a more detailed understanding of the resolution of orbifold singularities of non-Kähler geometries may reveal whether the resolution may allow gauge bundles with non-vanishing third Chern class.

\section{$3 \quad$ Orbifolding $T^{2}$-bundles over $K 3$}

New solutions can be constructed by orbifolding the $T^{2}$ bundle over $K 3$ geometry $X$ by a discrete symmetry group $\Gamma$ to obtain $Y=X / \Gamma$. We will discuss the characteristic features of such orbifolds in this section and give explicit models in the following sections.

When constructing orbifold models, we will in general only require that the physical fields $\left(g, H_{3}, \phi, F\right)$ remain invariant under the orbifold action $\Gamma$. FSY geometries are supersymmetric and thus solve the supergravity equations of motion which are written in terms of the physical fields. Thus, as long as the physical fields are invariant under $\Gamma$, the orbifold solution will also be a solution of the equations of motion.

However, the resulting orbifold solution will in general not be supersymmetric. Preserving supersymmetry will require additionally that the pair $(J, \Omega)$ is also invariant under $\Gamma$. As $J$ and $\Omega$ are bilinears of the spinor $\eta$ that 
generates $\mathcal{N}=1$ supersymmetry, e.g., $J_{m n}=i \eta^{\dagger} \gamma_{m n} \eta$ and $\Omega_{m n p}=\bar{\eta}^{\dagger} \gamma_{m n p} \eta$, a nowhere vanishing $\eta$ implies $(J, \Omega)$ must be globally well-defined on $Y$ and hence must be invariant on $X$ under the action of $\Gamma$.

The relations between the physical fields and $(J, \Omega)$ are given in $(2.4)$ to (2.6). From there, we see that $J$ must be invariant under $\Gamma$, since it is directly related to the physical metric. A central component of $J$ is $J_{K 3}$ which by uniqueness of the Calabi-Yau metric remains unchanged under $\Gamma$ as long as the discrete symmetry action leaves invariant the Kähler class and the complex structure of the base $K 3$. $\Omega$ however need not be invariant; the holomorphic (3,0)-form can transform by a phase, $\Gamma: \Omega \rightarrow \zeta \Omega$ for $|\zeta|=1$, and still leave $\phi$ invariant in (2.6) and the complex structure of $X$ unchanged. Thus, if $\zeta \neq 1$ then the resulting orbifold solution breaks all supersymmetry.

An element of the discrete symmetry group acting on $X$ can be thought of as being composed of two components: $\rho=\left(\rho_{1}, \rho_{2}\right)$, where $\rho_{1}$ acts on the $K 3$ base whereas $\rho_{2}$ acts on the torus fiber. It is useful to consider each action, $\rho_{1}$ and $\rho_{2}$, separately. On the $K 3$, finite group actions are standardly categorized by their actions on the holomorphic $(2,0)$-form. If an action leaves invariant $\Omega^{2,0}$, i.e., $\rho_{1}: \Omega^{2,0} \rightarrow \Omega^{2,0}$, then it is called a symplectic automorphism. If instead $\rho_{1}: \Omega^{2,0} \rightarrow \zeta \Omega$ for $\zeta \neq 1$, it is a called a non-symplectic automorphism. In general, the action $\rho_{1}$ on $K 3$ will have a non-empty fixed locus set which may consist of points and/or curves. On the other hand, the symmetry group action on the torus is much more limited. For a group of order $N$, we can have either a shift $\rho_{2}: z \rightarrow z+c$ for some complex constant $c$ such that $z+N c \sim z+n_{1}+n_{2} \tau$, or a rotation, $\rho_{2}: z \rightarrow \zeta z$ with $\zeta^{N}=1$.

Because the torus bundle is generically non-trivial and does not have a zero section, not any combination of $\left(\rho_{1}, \rho_{2}\right)$ will give a consistent symmetry action on $X$. Of importance, $X$ has a globally defined $(1,0)$-form $\theta=d z+\alpha$ which must have a well-defined transformation rule under $\Gamma$. We require for any $\rho \in \Gamma$

$$
\rho[\theta]=\rho_{2}[d z]+\rho_{1}[\alpha]=\zeta \theta .
$$

We see, for instance, that a rotation on the torus fiber by itself cannot be well defined on $X$. Consider the overlap region between two coordinate charts labelled by $A$ and $B$. The corresponding coordinates $z_{A}$ and $z_{B}$ and the local connection one-form $\alpha_{A}$ and $\alpha_{B}$ are related as follows:

$$
z_{A}=z_{B}+\varphi_{A B} \bmod 1, \tau, \quad \alpha_{A}=\alpha_{B}-d \varphi_{A B},
$$

where $\varphi_{A B}$ is the transition function with dependence on the local base $K 3$ coordinates. Thus, a rotation acting on $z$ (compatible with the torus lattice 
structure $\tau) \rho_{2}: z \rightarrow \zeta z$, only makes sense if there is a complementary action on the base $K 3$ such that the transition function transforms as $\rho_{1}: \varphi_{A B} \rightarrow$ $\zeta \varphi_{A B}$. By (3.2), we see that the connection $\alpha$ must then transform similarly with the phase $\zeta$ which thus results in a well-defined transformation phase for $\theta$. Condition (3.1) thus imposes a restriction on the allowable combination of $\left(\rho_{1}, \rho_{2}\right)$; and moreover, this restriction depends on the curvature $\omega=d \alpha$.

Let us now consider the distinguishing features of the orbifold solution for different types of symmetry group actions. We shall order them by the action on the torus fiber.

For $\rho_{2}$ that involves only a torus shift, the action is freely acting, i.e., without fixed points, and hence, the resulting orbifold geometry is always smooth. The amount of supersymmetry that is preserved depends on the action on $\Omega^{3,0}=\Omega^{2,0} \wedge \theta$. But since $\theta=d z+\alpha$ must be invariant under the torus shift (with $\alpha$ invariant under $\rho_{1}$ so that (3.1) is satisfied), the $\rho_{1}$ action on $\Omega^{2,0}$ determines whether supersymmetry is preserved. Thus, for symplectic $K 3$ automorphisms, $Y=X / \Gamma$ will preserve the supersymmetry of $X$ whereas the non-symplectic automorphism action will break all supersymmetry. The base action will, in general, have fixed points and/or fixed curves. From the fiber bundle description, the complex structure of the torus will jump along the fixed point locus of the base. Thus the torus bundle becomes a torus fibration. Clearly, $Y$ is topologically distinct from $X$ as the first fundamental group of $Y$ now contains $\Gamma$. Moreover, we note that the Betti numbers of $Y$ will generally differ from those of $X$ as the orbifolding by $\Gamma$ (with a non-trivial $\rho_{1}$ ) will project out non-invariant harmonic forms. Nevertheless, the Euler characteristic and $c_{3}(F)$ will remain zero as the orbifold is freely acting.

For the case where the torus fiber action involves a rotation, there should be an associated symmetry action on the base $K 3$ by (3.1). Moreover, the discrete symmetry group $\Gamma$ will, in general, have a fixed locus set which is invariant under $\left(\rho_{1}, \rho_{2}\right)$. The resulting orbifold geometry $Y$ will then be singular along the fixed points and/or fixed curves. These singularities will need to be resolved to obtain again a smooth solution. ${ }^{5}$ Here, the task of resolving singularities consists of two parts: resolving the manifold and smoothing out the physical fields such that the supersymmetry conditions (2.1) to (2.3) (or the supergravity equations of the motion) remain satisfied.

\footnotetext{
${ }^{5}$ Singularity resolution can be thought of as the geometrical analog of adding twisted sector states when constructing an orbifold conformal field theory.
} 
Resolving singular orbifolds $Y$ which are supersymmetric should follow the standard local toric resolutions of singular Calabi-Yau manifolds (see, e.g., [25]) by the requirement of a non-vanishing holomorphic $(3,0)$-form. However, unlike the Calabi-Yau case, where the vanishing of the first Chern class is the sole obstruction to the existence of a Calabi-Yau metric on a Kähler manifold, we do not presently know what are the obstructions or sufficient conditions for the existence of a solution to the heterotic supersymmetry differential constraints of (2.1) to (2.3). Without this, we must explicitly demonstrate the existence of solutions on a manifold on a case-bycase basis. Thus, the resolution of singularities in the non-Kähler scenario is a challenging mathematical problem. It requires constructing local models (such as [31]) that smooth out the singularities and carefully gluing the local geometries into $Y$.

Nevertheless, having orbifold singularities may lead to new non-Kähler solutions that might be phenomenologically appealing. For instance, in resolving the singularities, it may be possible to introduce local gauge bundles that have non-trivial $c_{3}(F)$, and hence non-zero number of generations. In essence, one may try to satisfy the requirements of low-energy phenomenology by inserting appropriate local models into the compact geometry. We shall, however, leave the subtleties of non-Kähler singularity resolutions for future work.

In the following sections, we will give concrete constructions of new heterotic solutions from orbifolding $X$ by discrete symmetries. This requires identifying those $K 3$ surfaces with a discrete symmetry group action. We will consider three types of $K 3$ surfaces that naturally contain discrete symmetries: $K 3$ surfaces of Kummer type, $K 3$ surfaces with a branched covering description, and more general algebraic (i.e., projective) $K 3$ surfaces with finite group actions.

\section{Orbifold limit of Kummer $K 3$ base}

In this section, we construct new orbifold geometries starting from those FSY geometries with a Kummer $K 3$ base. A Kummer $K 3$ has a $T^{4} / \mathbb{Z}_{2}$ orbifold limit with the involution (i.e., $\mathbb{Z}_{2}$ action) given by

$$
\sigma:\left(z_{1}, z_{2}\right) \rightarrow\left(-z_{1},-z_{2}\right)
$$

where $\left(z_{1}, z_{2}\right)$ are the complex coordinates of $T^{4}$. The smooth Kummer surface is obtained by blowing-up each of the 16 singular fixed points of the involution $\sigma$ with a $\mathbb{P}^{1}$. Away from the singularities, the hermitian metric 
and holomorphic $(3,0)$-form take the simple form

$$
\begin{aligned}
& J=\frac{\mathrm{i}}{2} \mathrm{e}^{2 \phi}\left(d z_{1} \wedge d \bar{z}_{1}+d z_{2} \wedge d \bar{z}_{2}\right)+\mathrm{i} \frac{A}{2} \theta \wedge \bar{\theta} \\
& \Omega=d z_{1} \wedge d z_{2} \wedge \theta
\end{aligned}
$$

where $\phi$ is the dilaton field and $\theta=d z+\alpha$ denotes the one-form associated to the twisted torus fiber. For simplicity, we have set the complex structure of the three covering-space tori to be $\tau=\mathrm{i}$. Thus for the base coordinates $\left(z_{1}, z_{2}\right)$ and fiber coordinate $z$, we identify $z_{i} \sim z_{i}+1 \sim z_{i}+i$, for $i=1,2$, and $z \sim z+1 \sim z+\mathrm{i}$. Given a torus twist curvature $\omega$, we can then set the torus fiber area $A$ to satisfy the topological condition (2.11).

We shall work mainly in the $T^{4} / \mathbb{Z}_{2}$ orbifold limit of the Kummer $K 3{ }^{6}$ This simplification will allow us to write down explicitly the discrete symmetry group $\Gamma$ acting on $X$ and demonstrate clearly some of the characteristics of the new orbifold geometries discussed in Section 3. The discrete symmetry groups $\Gamma$ that we consider are all cyclic and thus are generated by a single element which we will denote by $\rho$.

\section{$4.1 \mathcal{N}=1,2$ supersymmetric orbifolds}

Different types of orbifold actions will leave unbroken different amounts of supersymmetry in four dimensions. We consider first those orbifold actions that leave invariant the holomorphic $(3,0)$-form and preserve the supersymmetry of the covering FSY geometry. We will give four examples below and will point out the distinctive features of each.

Example 1. Freely acting with a shift action on the torus fiber.

We start with a simple model that has no fixed points, and therefore has a vanishing Euler characteristic and a vanishing number of generations. Consider the $\mathbb{Z}_{2}$ action generated by

$$
\rho:\left(z_{1}, z_{2}, z\right) \rightarrow\left(\mathrm{i} z_{1},-\mathrm{i} z_{2}, z+\frac{1}{2}\right)
$$

which rotates the base in an $\mathrm{SU}(2.2)$ invariant manner and shifts the torus fiber. For the above discrete identifications, it is easy to see that on the

\footnotetext{
${ }^{6}$ Some earlier discussion on torsional orbifolds obtained via duality from Borcea fourfolds in M-theory appeared in [18].
} 
four-dimensional base, the $\mathbb{Z}_{2}$ action has four fixed points located at

$$
\left(z_{1}, z_{2}\right)=\left\{(0,0),\left(0, \frac{1+\mathrm{i}}{2}\right),\left(\frac{1+\mathrm{i}}{2}, 0\right),\left(\frac{1+\mathrm{i}}{2}, \frac{1+\mathrm{i}}{2}\right)\right\} .
$$

But since the torus fiber is concurrently shifted by $z \rightarrow z+1 / 2$, the sixdimensional quotient manifold does not have any singularities and is thus smooth.

The above background is supersymmetric because, as follows from (4.2), the pair $(J, \Omega)$ is invariant under $\rho$ as long as $\theta=d z+\alpha$ is invariant. This can be easily satisfied by choosing a curvature twist $\omega=d \theta \in H^{(2,0)} \oplus H^{(1,1)}$ $\left(T^{4} / \mathbb{Z}_{2}, \mathbb{Z}\right)$ that is primitive with respect to the base and invariant under $\rho$. For example, $\omega$ can be any linear combination of $d z_{1} \wedge d z_{2}$ and $d z_{1} \wedge d \bar{z}_{1}-$ $d z_{2} \wedge d \bar{z}_{2}$. As shown in [28], theories with a twist of type $(1,1)$ have $\mathcal{N}=2$ supersymmetry whereas a more general twist of type $(2,0)+(1,1)$ leads to a theory with $\mathcal{N}=1$ supersymmetry. Similarly, for the gauge field, we can choose $U(1)$ gauge field strengths $F=F^{(1,1)}$ which are invariant under $\rho$. This then ensures that the three supersymmetry constraint equations (2.1) to (2.3) are invariant under $\rho$. This background also naturally satisfies the equations of motion since the physical fields $\left(g_{a \bar{b}}, H, \phi, F_{a \bar{b}}\right)$ are invariant under the orbifold action.

As a fiber space, this smooth quotient geometry should be considered as a $T^{2}$ fibration rather than a $T^{2}$ bundle as the complex structure of the $T^{2}$ fiber jumps at the four singular points (4.4) of the base identified by the involution action $\rho$. At the fixed points, the complex structure jumps to $\tau=2 \mathrm{i}$ with $z \sim z+\frac{1}{2} \sim z+\mathrm{i}$.

Finally, we note also that the $\mathbb{Z}_{2}$ action (4.3) preserves not only the holomorphic $(3,0)$-form but also the holomorphic $(2,0)$-form of the base $K 3$. Its action on the base $K 3$ is thus an example of a symplectic automorphism, a discrete symmetry group that preserve $\Omega^{2,0}$.

Example 2. Freely acting with a reflection on the torus fiber.

It is also possible to consider a freely acting involution which involves a non-symplectic automorphism, one that acts non-trivially on the holomorphic $(2,0)$-form on the base $K 3$. One such involution is generated by

$$
\rho:\left(z_{1}, z_{2}, z\right) \rightarrow\left(-z_{1}+\frac{1}{2}, z_{2}+\frac{1}{2},-z\right) .
$$

$\rho$ here acts freely on the base coordinates $\left(z_{1}, z_{2}\right)$, and has been called an Enriques involution [32] since the base holomorphic $(2,0)$-form is mapped 
to the minus of itself

$$
\rho: \Omega_{K 3} \rightarrow-\Omega_{K 3} .
$$

The holomorphic $(3,0)$-form, however, remains invariant when we take into account the compensating reflection action, $z \rightarrow-z$, on the fiber coordinate.

Precisely the involution in (4.5) acting on the product space $K 3 \times T^{2}$, was analyzed in $[33,34]$ in the context of type IIA/heterotic string duality. That such a quotient can also be applied to the FSY geometry with a nontrivial principal $T^{2}=S^{1} \times S^{1}$ bundle with no zero section may at first seem surprising. But as explained earlier, as long as we carefully choose a torus twist that gets reflected along with the fiber coordinate such that the oneform $\theta=d z+\alpha$ has a well-defined action, then the $\mathbb{Z}_{2}$ action (4.5) consisting of a fiber reflection coupled with an involution on the base is well defined. Let us verify this explicitly for this example.

First, a consistent torus twist for the $\mathbb{Z}_{2}$ action (4.5) is

$$
\theta=d z+A_{1}\left(z_{1}-\bar{z}_{1}\right) d z_{2}+A_{2}\left(z_{2}-\bar{z}_{2}\right) d z_{1},
$$

where $A_{1}$ and $A_{2}$, are Gaussian integers (complex numbers with integral real and imaginary parts). Note that with this one form, the torus twist curvature $\omega=d \theta$ contains both a $(1,1)$ and a $(2,0)$ part so the covering FSY geometry and also the orbifold geometry preserve only $\mathcal{N}=1$ supersymmetry.

With the one-form (4.7), let us demonstrate explicitly the consistency of the quotient action. We will work in the covering space which is a $T^{2}$ bundle over a $T^{4}$ base. The metric on this space (neglecting the warp factor and the torus area $A$ which do not a play a role here) takes the form

$$
d s^{2}=\left|d z_{1}\right|^{2}+\left|d z_{2}\right|^{2}+\left|d z+A_{1}\left(z_{2}-\bar{z}_{2}\right) d z_{1}+A_{2}\left(z_{1}-\bar{z}_{1}\right) d z_{2}\right|^{2} .
$$

In order for the metric to be well defined, the local complex coordinates must have the periodicity

$$
\begin{aligned}
z_{1} & \sim z_{1}+a, \\
z_{2} & \sim z_{2}+b, \\
z & \sim z+c-A_{1}(b-\bar{b}) z_{1}-A_{2}(a-\bar{a}) z_{2},
\end{aligned}
$$

where $a, b, c$ are arbitrary Gaussian numbers. These periodic boundary conditions define the transition functions on the manifold. The quotient action 
(4.5) acts on the periodicity (4.9) and results in

$$
\begin{aligned}
-z_{1} & \sim-z_{1}+a=-\left(z_{1}+a^{\prime}\right), \\
z_{2} & \sim z_{2}+b=z_{2}+b^{\prime}, \\
-z & \sim-z+c+A_{1}(b-\bar{b})\left(z_{1}-\frac{1}{2}\right)-A_{2}(a-\bar{a})\left(z_{2}+\frac{1}{2}\right) \\
& =-\left(z+c^{\prime}-A_{1}\left(b^{\prime}-\bar{b}^{\prime}\right) z_{1}-A_{2}\left(a^{\prime}-\bar{a}^{\prime}\right) z_{2}\right),
\end{aligned}
$$

where we have defined

$$
\left(a^{\prime}, b^{\prime}, c^{\prime}\right)=\left(-a, b,-c+\mathrm{i} A_{1} \operatorname{Im}(b)+\mathrm{i} A_{2} \operatorname{Im}(a)\right) .
$$

Since the constants $a, b, c$ are arbitrary Gaussian numbers, the redefinition of (4.11) is inconsequential. Therefore, from (4.10), we see that the quotient action preserves the periodicity (4.9) of the covering space. This implies that the quotient is well-defined.

Example 3. An involution with fixed curves.

From the previous example, we have seen how a discrete symmetry action can involve a reflection, a simple example of a rotation action on the fiber. Coupled with the discrete symmetry action on the base, the discrete group action with fiber rotation action will generally have fixed points and/or fixed curves.

Consider the $\mathbb{Z}_{2}$ action generated by

$$
\rho:\left(z_{1}, z_{2}, z\right) \rightarrow\left(\mathrm{i} z_{1}, \mathrm{i} z_{2},-z\right) .
$$

This is an involution since $(\rho)^{2}$ is equivalent to $\sigma$, the $\mathbb{Z}_{2}$ action appearing in (4.1). This involution again involves a reflection on the $T^{2}$ bundle. As above we need to require that the curvature twist transforms $\omega \rightarrow-\omega$ under $\rho$. For this we must have $\omega=d \theta \sim d z_{1} \wedge d z_{2}$ and this will ensure that the one-form $\theta$ is well-defined under the involution.

The quotient manifold however will have fixed curves. On the $T^{6}$ covering space, the $\sigma$ and $\rho$ actions result in 16 fixed points - four fixed points on the base times and four fixed points on the fiber. The four base fixed points (those of (4.4)) coincide with the singularities of the $T^{4} / \mathbb{Z}_{2}$ orbifold and are resolved by $\mathbb{P}^{1}$ 's when the base $K 3$ orbifold is resolved. These $\mathbb{P}^{1}$ 's however are invariant under $\rho$ and result in 16 fixed curves.

We can explicitly see this, for instance, in the resolution of the point $\left(z_{1}, z_{2}, z\right)=(0,0,0)$. Locally, this is equivalent to resolving the origin of 
$C^{3} /\left(\mathbb{Z}_{2} \times \mathbb{Z}_{2}\right)$ with the quotient generated by $\sigma$ and $\rho$. This local orbifold can be minimally resolved following the toric resolution methods of $[35,36]$. We resolve the singularity in two steps. We first resolve the singularity at the origin of $C^{2} /\{1, \sigma\} \times C$ and then proceed to quotient by $\rho$ and resolve again.

For $C^{2} /\{1, \sigma\} \times C$, the origin is resolved by a $\mathbb{P}^{1}$. Following e.g., [35], the resolution is covered by two coordinate charts:

$$
\begin{array}{ll}
U_{1}: & \left(\frac{z_{2}}{z_{1}}, z_{1}^{2}, z\right), \\
U_{2}: & \left(\frac{z_{1}}{z_{2}}, z_{2}^{2}, z\right) .
\end{array}
$$

Here, the first entry is the coordinate of the $\mathbb{P}^{1}: \xi=\frac{z_{2}}{z_{1}}$ in $U_{1}$ and $\xi^{\prime}=1 / \xi$ in $U_{2}$. We can proceed to apply the $\rho$ action (4.12) on the two coordinate charts:

$$
\begin{array}{ll}
U_{1}: & \rho:\left(\frac{z_{2}}{z_{1}}, z_{1}^{2}, z\right) \rightarrow\left(\frac{z_{2}}{z_{1}},-z_{1}^{2},-z\right), \\
U_{2}: & \rho: \quad\left(\frac{z_{1}}{z_{2}}, z_{2}^{2}, z\right) \rightarrow\left(\frac{z_{1}}{z_{2}},-z_{2}^{2},-z\right) .
\end{array}
$$

We see that $\rho$ leaves the $\mathbb{P}^{1}$ coordinate $\xi$ (and $\xi^{\prime}$ ) invariant and acts only on the two transverse coordinates. This shows that the $\mathbb{P}^{1}$ curve is invariant under the $\rho$ action. To resolve the two transverse coordinates, which is another $C^{2} /\{1, \sigma\}$ singularity, we can repeat the resolution of (4.13) on $U_{1}$ and $U_{2}$ charts separately. The total resolution is therefore described by four coordinate charts: $U_{11}, U_{12}, U_{21}, U_{22}$ :

$$
\begin{aligned}
& U_{11}: \quad\left(\frac{z_{2}}{z_{1}}, \frac{z}{z_{1}^{2}}, z_{1}^{4}\right), \quad U_{21}: \quad\left(\frac{z_{1}}{z_{2}}, \frac{z}{z_{2}^{2}}, z_{2}^{4}\right), \\
& U_{12}: \quad\left(\frac{z_{2}}{z_{1}}, \frac{z_{1}^{2}}{z}, z^{2}\right), \quad U_{22}: \quad\left(\frac{z_{1}}{z_{2}}, \frac{z_{2}^{2}}{z}, z^{2}\right) .
\end{aligned}
$$

On every point of the $\mathbb{P}^{1}$ curve, we have added another $\mathbb{P}^{1}$. Thus the divisor is a ruled surface, and by the fibration structure, it is a Hirzebruch $F_{2}$ surface which has Euler characteristic $\chi\left(F_{2}\right)=4$. After resolving all $16 \mathbb{P}^{1}$ 's, the Euler characteristic of the resolved orbifold $\widehat{Y}$ is

$$
\chi(\widehat{Y})=\left[\chi(X)-16 \chi\left(\mathbb{P}^{1}\right)\right] / 2+16 \chi\left(F_{2}\right)=-16+64=48 .
$$

We have thus described how to resolve the singular orbifold into a smooth manifold with non-zero Euler characteristic. If we are only interested in 
Calabi-Yau solutions, then this is sufficient to describe the solution as Yau's theorem implies the existence of a Ricci-flat metric. However, without a corresponding theorem for the existence of non-Kähler heterotic solutions, we have to demonstrate explicitly that a non-Kähler balanced metric exists for this resolved manifold. This may be done by constructing non-compact solutions that locally resolved the singularities. One would then need to cut out the singularities and carefully glue in these local solutions into the manifold.

Example 4. A $\mathbb{Z}_{4}$ quotient with fixed points and fixed curves singularities.

In general, the generic orbifold model will have both fixed points and fixed curves singularities which will need to be resolved. Let us give an example.

Consider the $\mathbb{Z}_{4}$ action generated by

$$
\rho:\left(z_{1}, z_{2}, z\right) \rightarrow\left(\mathrm{i} z_{1},-z_{2}, \mathrm{i} z\right) .
$$

Though very similar to the previous example, this action does not square to the $\mathbb{Z}_{2}$ identification (4.1) of the $K 3$. Thus it is a $\mathbb{Z}_{4}$ action. In order for $\theta$ to be well defined, we are constrained to require that the torus curvature twist transforms as $\omega \rightarrow \mathrm{i} \omega$, which implies that $\omega \sim d \bar{z}_{1} \wedge d z_{2}$.

The action (4.17) has eight fixed points on the base given by

$$
\begin{aligned}
\left(z_{1}, z_{2}\right)= & \left\{(0,0),\left(0, \frac{1}{2}\right),\left(0, \frac{\mathrm{i}}{2}\right),\left(0, \frac{1+\mathrm{i}}{2}\right)\left(\frac{1+\mathrm{i}}{2}, 0\right),\left(\frac{1+\mathrm{i}}{2}, \frac{1}{2}\right),\right. \\
& \left.\left(\frac{1+\mathrm{i}}{2}, \frac{\mathrm{i}}{2}\right),\left(\frac{1+\mathrm{i}}{2}, \frac{1+\mathrm{i}}{2}\right)\right\}
\end{aligned}
$$

and two fixed points on the fiber at $z=\left\{0, \frac{1+i}{2}\right\}$. The base fixed points again coincide with those of the orbifold $T^{4} / \mathbb{Z}_{2}$ and are resolved by $\mathbb{P}^{1}$ 's. However, these $\mathbb{P}^{1}$ 's are not invariant under $\rho$ as defined in (4.17). We can see this from the resolution of the point $(0,0,0)$. The action of $\rho$ on the two charts of $\mathbb{P}^{1}$ is

$$
\begin{aligned}
& U_{1}: \quad \rho: \quad\left(\frac{z_{2}}{z_{1}}, z_{1}^{2}, z\right) \rightarrow\left(\mathrm{i} \frac{z_{2}}{z_{1}},-z_{1}^{2}, \mathrm{i} z\right), \\
& U_{2}: \quad \rho: \quad\left(\frac{z_{1}}{z_{2}}, z_{2}^{2}, z\right) \rightarrow\left(-\mathrm{i} \frac{z_{1}}{z_{2}}, z_{2}^{2}, \mathrm{i} z\right) .
\end{aligned}
$$

We see that $\rho$ acts differently on the two charts. But in each case, the resolved $\mathbb{P}^{1}$, denoted by the first coordinate, is also rotated by the $\rho$ action. So considering the base and fiber together, we have here 16 true fixed points. 
There are also fixed curves in this model. They arise from points that are fixed under $\rho^{2}$ but not $\rho$. The $\rho^{2}$ action, $\rho^{2}:\left(z_{1}, z_{2}, z\right) \rightarrow\left(-z_{1}, z_{2},-z\right)$, has fixed curves at

$$
\begin{aligned}
\left(z_{1}, z\right)=\{ & \left(0, \frac{1}{2}\right),\left(\frac{1+i}{2}, \frac{1}{2}\right),\left(\frac{1}{2}, 0\right),\left(\frac{1}{2}, \frac{1}{2}\right),\left(\frac{1}{2}, \frac{\mathrm{i}}{2}\right),\left(\frac{1}{2}, \frac{1+\mathrm{i}}{2}\right), \\
& \left.\left(0, \frac{\mathrm{i}}{2}\right),\left(\frac{1+\mathrm{i}}{2}, \frac{\mathrm{i}}{2}\right),\left(\frac{\mathrm{i}}{2}, 0\right),\left(\frac{\mathrm{i}}{2}, \frac{\mathrm{i}}{2}\right),\left(\frac{\mathrm{i}}{2}, \frac{1}{2}\right),\left(\frac{\mathrm{i}}{2}, \frac{1+\mathrm{i}}{2}\right)\right\} .
\end{aligned}
$$

These curves are not invariant under $\rho$; in fact, the curves on the first line of (4.20) are identified with those on the second line in the local coordinates $\left(z_{1}, z\right)$. For $z_{2} \neq 0, \frac{1}{2}, \frac{\mathrm{i}}{2}, \frac{1+\mathrm{i}}{2}$ which are the fixed points of $z_{2}$ under $\rho$, the 12 curves persist. At the $z_{2}$ fixed points, the identification reduces to six distinct curves.

\subsection{Non-supersymmetric orbifolds}

Non-supersymmetric models can easily be constructed similar to the examples given above. The orbifold action now is required to act on the holomorphic three-form non-trivially. Consider the $\mathbb{Z}_{2}$ action generated by

$$
\rho:\left(z_{1}, z_{2}, z\right) \rightarrow\left(\mathrm{i} z_{1}, \mathrm{i} z_{2}, z+1 / 2\right) .
$$

Here, $\rho$ differs from the action in (4.3) by a minus sign in the action on $z_{2}$. As a result, the base holomorphic two-form $\Omega_{T^{4} / \mathbb{Z}_{2}}=d z_{1} \wedge d z_{2}$ picks up a minus sign and the holomorphic three-form transforms non-trivially, i.e., $\Omega \rightarrow-\Omega$ and is thus projected out. Therefore, this type of solution is not supersymmetric.

The torus curvature $\omega=d \theta$ and $U(1)$ field strength $F$ are required to be primitive and invariant under $\rho$. A basis of such $(1,1)$ forms is $\left\{d z_{1} \wedge\right.$ $\left.d \bar{z}_{2}, d z_{2} \wedge d \bar{z}_{1}, d z_{1} \wedge d \bar{z}_{1}-d z_{2} \wedge d \bar{z}_{2}\right\}$. All the physical fields $\left(g, H_{3}, \phi, F\right)$ of the model can remain invariant under $\rho$. Thus, this gives a smooth nonsupersymmetric solution of the supergravity equations of motion.

\section{Orbifolds from branched covered $K 3$ base}

We are interested in complex manifolds whose first Chern class $c_{1}(M)$ is zero. When the canonical bundle is non-trivial, i.e., $c_{1}(M) \neq 0$, it is sometimes 
possible to eliminate the first Chern class by taking $n$ copies (aka covers) of the manifold $M$ and glue them together at a divisor, a codimension one hypersurface. More precisely, this new manifold $M^{\prime}$, which is described as an $n$-fold cover of $M$ branched over a divisor $D$, can have trivial canonical bundle. $^{7}$ The new Chern class is given by

$$
c_{1}\left(M^{\prime}\right)=n\left[c_{1}(M)-c_{1}(D)\right]+c_{1}(D)=n c_{1}(M)-(n-1) c_{1}(D) .
$$

A Calabi-Yau manifold with an $n$-fold branched covering description clearly has a manifest $\mathbb{Z}_{n}$ discrete symmetry acting on the $N$ identical covers. In this section, we construct orbifold solutions starting from FSY geometries containing a $K 3$ base with a branched covering description. Below, we will make use of two examples of branched covered $K 3$ surfaces: a triple cover of $\mathbb{P}^{1} \times \mathbb{P}^{1}$ branched over a genus four curve and a double cover of $\mathbb{P}^{2}$ branched over a genus 10 curve.

Before jumping into the details of branched covered $K 3$ surfaces, let us first illustrate the basic idea of using branched covering for the simpler complex dimension one case where the first Chern number agrees with the Euler characteristic $C_{1}(M)=\chi(M)$. Let $M=\mathbb{P}^{1}=S^{2}$. Being in two real dimensions, $\chi_{1}\left(M^{\prime}\right)=0$ means $M^{\prime}=T^{2}$ for compact manifolds. Since $\chi_{1}(M)=2$ and $\chi_{1}(D)$ equals the number of branched points, we see from (5.1) that one can construct a torus as a two-fold cover of $\mathbb{P}^{1}$ branched over four points or as a three-fold cover of $\mathbb{P}^{1}$ branched over three points. Reversing the construction, we can start with the double covered torus $M^{\prime}=T^{2}$ and quotient by an involution to obtain a $\mathbb{P}^{1}$ with four fixed points, or with the triple covered torus and quotient by a $\mathbb{Z}_{3}$ action to obtain a $\mathbb{P}^{1}$ with three fixed points. Such natural quotienting can be applied similarly to branched covered $K 3$ surfaces.

\section{$5.1 \quad \mathbb{P}^{1} \times \mathbb{P}^{1}$ base solution}

We take the base $K 3$ surface to be a triple cover of $\mathbb{P}^{1} \times \mathbb{P}^{1}$ branched over a sextic curve. As described below, this $K 3$ is a complete intersection of a quadric and a cubic equation in $\mathbb{P}^{4}$. Using this $K 3$ to construct a FSY geometry, we can quotient by the natural $\mathbb{Z}_{3}$ action and obtain an orbifold non-Kähler heterotic solution that is an elliptic fibration over a $\mathbb{P}^{1} \times \mathbb{P}^{1}$ base.

\footnotetext{
${ }^{7}$ For examples of Calabi-Yau three-fold with a branched covering description, see [21-23].
} 
To describe the $K 3$, let $\left\{z_{0}, z_{1}, z_{2}, z_{3}, z_{4}\right\}$ be the homogeneous coordinates of $\mathbb{P}^{4}$. The $K 3$ hypersurface in $\mathbb{P}^{4}$ is defined by the following two equations:

$$
\begin{gathered}
f^{1}=z_{0} z_{3}-z_{1} z_{2}=0, \\
f^{2}=g\left(z_{0}, z_{1}, z_{2}, z_{3}\right)+z_{4}^{3}=0,
\end{gathered}
$$

where $g$ is a degree three homogeneous polynomial in $z_{i}$. The first equation enforces the standard embedding of $\mathbb{P}^{1} \times \mathbb{P}^{1} \rightarrow \mathbb{P}^{3} \subset \mathbb{P}^{4}$ by the mapping

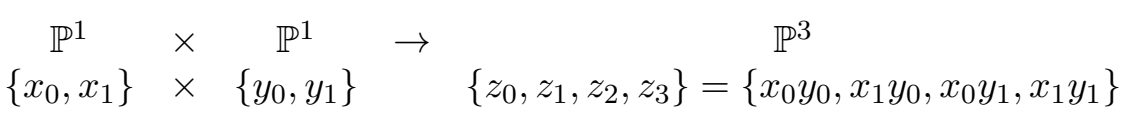

The second equation exhibits the three-fold covering. For each generic point on $\mathbb{P}^{1} \times \mathbb{P}^{1}$, there are three different values of $z_{4}$ that satisfy (5.2). Alternatively, the equations above are invariant under the $\mathbb{Z}_{3}$ action generated by

$$
\rho: z_{4} \rightarrow \zeta z_{4}, \quad \text { where } \quad \zeta=e^{2 \pi \mathrm{i} / 3}
$$

In particular, the special point $z_{4}=0$ is invariant under this action and here we have the branched curve specified by

$$
g\left(z_{0}, z_{1}, z_{2}, z_{3}\right)=0
$$

This is a cubic equation in terms of the $z_{i}$ variables and a sextic equation in terms of the natural homogeneous variables $x_{i}, y_{i}$ on $\mathbb{P}^{1} \times \mathbb{P}^{1}$. To ensure the hypersurface is smooth, it is sufficient to require that the normal bundle to the hypersurface does not vanish. That is

$$
d f^{1} \wedge d f^{2}=\left(z_{3} d z_{0}-z_{2} d z_{1}-z_{1} d z_{2}+z_{0} d z_{3}\right) \wedge\left(\sum_{i=0}^{3} \partial_{i} g d z_{i}+3 z_{4}^{2} d z_{4}\right) \neq 0,
$$

for any points on the hypersurface. This is a constraint for $g$. For instance, it can be checked that a hypersurface defined by $g=z_{0}^{3}+z_{1}^{3}+z_{2}^{3}+z_{3}^{3}$ is singular for example at $\left(z_{0}, z_{1}, z_{2}, z_{3}\right)=(1,-1,-1,1)$ while $g=z_{0}^{3}+z_{1}^{3}+$ $z_{2}^{3}+2 z_{3}^{3}$, the example we shall consider below, is everywhere smooth.

We can write down explicitly the holomorphic two-form for the $K 3$ hypersurface. (For reference, see, e.g., [37, Section 15.4].) In the local chart where $z_{0} \neq 0$, we define the affine coordinates $Z_{i}=z_{i} / z_{0}$ for $i=0, \ldots, 3$. In these 
coordinates the constraint polynomials become

$$
\begin{aligned}
& f^{1}=Z_{3}-Z_{1} Z_{2}=0, \\
& f^{2}=g\left(1, Z_{1}, Z_{2}, Z_{3}\right)+Z_{4}^{3}=0 .
\end{aligned}
$$

The holomorphic two form in terms of $d Z_{1} \wedge d Z_{2}$ is then

$$
\Omega^{2,0}=d Z_{1} \wedge d Z_{2} /(\operatorname{det} M)=\frac{d Z_{1} \wedge d Z_{2}}{3 Z_{4}^{2}},
$$

where the $2 \times 2$ matrix $M_{i j}=\partial f^{i} / \partial Z_{2+j}$ consists of the partial derivatives of the two other coordinates $\left(Z_{3}, Z_{4}\right)$. The choice of the $\left(Z_{1}, Z_{2}\right)$, of course, is arbitrary and we could have used any other two coordinates. For instance, in terms of $d Z_{3} \wedge d Z_{4}$, we have

$$
\Omega^{2,0}=\frac{d Z_{3} \wedge d Z_{4}}{Z_{1} \partial_{1} g-Z_{2} \partial_{2} g} .
$$

When (5.7) is satisfied for a smooth $K 3$ hypersurface, the different det $M$ for different choices of coordinates never simultaneously vanish. This must be the case since $\Omega^{2,0}$ is no-where vanishing. Moreover, of importance for our construction, under the $\mathbb{Z}_{3}$ action, $\rho: Z_{4} \rightarrow \zeta Z_{4}$, the holomorphic two form is not invariant and in fact transforms as $\Omega^{2,0} \rightarrow \zeta \Omega^{2,0}$. This is clearly evident in (5.10) and (5.11). In contrast, the Calabi-Yau metric or the hermitian form $J_{K 3}$ is invariant under $\mathbb{Z}_{3}$.

We now consider the non-singular example with the $K 3$ hypersurface defined by

$$
\begin{aligned}
f^{1} & =g\left(z_{0}, z_{1}, z_{2}, z_{3}\right)+z_{4}^{4}=z_{0}^{3}+z_{1}^{3}+z_{2}^{3}+2 z_{3}^{3}+z_{4}^{3}, \\
& =\left(x_{0}^{3}+x_{1}^{3}\right)\left(y_{0}^{3}+y_{1}^{3}\right)+x_{1}^{3} y_{1}^{3}+z_{4}^{3}=0,
\end{aligned}
$$

where we have substituted in the coordinates of the two $\mathbb{P}^{1}$ 's, $x_{i}$ and $y_{i}$. The branched curve $C$ is located at $g=z_{4}=0$. Assuming $z_{0}=x_{0} y_{0} \neq 0$, the equation of the branched curve can be written as

$$
\left(1+X^{3}\right)\left(1+Y^{3}\right)+X^{3} Y^{3}=-Z_{4}^{3}=0,
$$

where $X=x_{1} / x_{0}$ and $Y=y_{1} / y_{0}$. (Note from the relation $\chi(K 3)=3\left[\chi\left(\mathbb{P}^{1} \times\right.\right.$ $\left.\left.\mathbb{P}^{1}\right)-\chi(C)\right]+\chi(C)$, we see that branched curve has genus $g=4$.) Two 
distinguished curves in this model are obviously the curves on each of the $\mathbb{P}^{1}$. These follow from (5.13) by taking either $X$ or $Y$ to be fixed

$$
\begin{array}{ll}
C_{X^{\prime}}: & -Z_{4}^{3}=1+Y^{3}+X^{\prime 3}\left(1+2 Y^{3}\right), \\
C_{Y^{\prime}}: & -Z_{4}^{3}=1+X^{3}+Y^{\prime 3}\left(1+2 X^{3}\right),
\end{array}
$$

where $X^{\prime}, Y^{\prime} \in \mathbf{C}$ are complex constants. Note that the $C_{X^{\prime}}$ and $C_{Y^{\prime}}$ curves are holomorphically embedded into the $K 3$ surface. The two classes are also homologously inequivalent. The class of $C_{X^{\prime}}$ curves do not self-intersect whereas they intersect three times with the $C_{Y^{\prime}}$ curves.

Given the above $K 3$ surface, we can build a FSY geometry. We twist the $T^{2}$ by a curvature $\omega=\omega_{1}-\omega_{2}$, where $\omega_{1}$ and $\omega_{2}$ are the two forms dual to the curves $C_{X^{\prime}}$ and $C_{Y^{\prime}}$. The explicit expressions for these forms can be obtained by Poincaré duality though we will not need them. Notice that $\omega=\omega_{1}-\omega_{2}$ is primitive with respect to the Kähler form on $\mathbb{P}^{1} \times \mathbb{P}^{1}$ and also $J_{K 3}$. Moreover, since the curves $C_{X^{\prime}}$ and $C_{Y^{\prime}}$ are holomorphically embedded, $\omega \in H^{1,1}(K 3) \cap H^{2}(K 3, \mathbb{Z})$. $\omega$ can also be the curvature form of any $U(1)$ gauge bundles that one wish to turn on.

This construction provides an explicit algebraic description of the covering FSY geometry. We can now orbifold by a $\mathbb{Z}_{3}$ action to obtain a new solution. Again, we can take the fiber torus to have square periodicities $z \sim z+1 \sim$ $z+\mathrm{i}$. The $\mathbb{Z}_{3}$ action acting on the six-dimensional geometry is generated by

$$
\rho:\left\{z_{0}, z_{1} . z_{2}, z_{3}, z_{4}, z\right\} \rightarrow\left\{z_{0}, z_{1}, z_{2}, z_{3}, \zeta z_{4}, z+1 / 3\right\}
$$

where $\zeta$ is a third root of unity, i.e., $\zeta^{3}=1 . \rho$ acting on the base reduces the triple cover to just a single copy of $\mathbb{P}^{1} \times \mathbb{P}^{1}$. Without the $T^{2}$ bundle, we have a singular branched curve that is invariant under $\rho$. The shift action on $T^{2}$ removes the singular identification; however, it also increases the complex structure $\tau$ of the $T^{2}$ fiber along the branched curve. The torus complex structure thus jumps along the branched curve. But nevertheless, the resulting geometry is smooth since there are no fixed points.

One might worry about the $\mathbb{Z}_{3}$ action on the curvature $\omega$. But since $\omega$ is dual to the curves, $C_{1}$ and $C_{2}$, which are invariant under $\rho, \omega$ must also be invariant under $\rho$.

The FSY geometry with the three-fold cover $K 3$ surface is thus invariant under $\rho$. Since the holomorphic three-form $\Omega=\Omega^{2,0} \wedge \theta$ transforms nontrivially $\rho: \Omega \rightarrow \zeta \Omega$, the smooth orbifold solution with an elliptic fibration over $\mathbb{P}^{1} \times \mathbb{P}^{1}$ is non-supersymmetric. 


\section{$5.2 \quad \mathbb{P}^{2}$ base solution}

We can take the $K 3$ surface to be a double cover over a $\mathbb{P}^{2}$ base branched over a sextic curve. The $K 3$ surface is defined as a degree six hypersurface in $W \mathbb{P}_{1113}^{3}$. With weighted homogeneous coordinates $\left(z_{0}, z_{1}, z_{2}, z_{3}\right)$, we can take the hypersurface to be

$$
z_{0}^{6}+z_{1}^{6}+z_{2}^{6}+z_{3}^{2}=0 .
$$

Notice that $\left(z_{0}, z_{1}, z_{2}\right)$ defines a $\mathbb{P}^{2}$. And for each point on $\mathbb{P}^{2}$, there are two values of $z_{3}$ that satisfy (5.16), except on the degree six (genus ten) branched curve

$$
g\left(z_{0}, z_{1}, z_{2}\right)=z_{0}^{6}+z_{1}^{6}+z_{2}^{6}=0 .
$$

Similar to the $\mathbb{P}^{1} \times \mathbb{P}^{1}$ case, the $\Omega^{2,0}$ form on the base is not invariant under the $\mathbb{Z}_{2}$ action $z_{3} \rightarrow-z_{3}$. However, we can preserve the holomorphic three form by considering the $\mathbb{Z}_{2}$ quotient generated by

$$
\rho:\left(z_{3}, z\right) \rightarrow\left(-z_{3},-z\right) .
$$

Taking the twist curvature $\omega \sim \Omega^{2,0}$, ensures that $\theta \rightarrow-\theta$ under $\rho . \Omega=$ $\Omega^{2,0} \wedge \theta$ is therefore invariant under the $\mathbb{Z}_{2}$ action. The degree six branched curve defined by (5.17) is however singular. Thus, this construction gives a singular $\mathcal{N}=1$ supersymmetric orbifold solution. As before, we leave the resolution of these singular curves for future work.

\section{Algebraic $K 3$ surfaces with finite group action}

In the previous section, we have seen two examples of $K 3$ surfaces with a branched covering description and with it a natural discrete symmetry group action. In both cases, the discrete symmetries are non-symplectic automorphisms which by definition act non-trivially on the holomorphic two-form $\Omega^{2,0}$ of the $K 3$. More generally, $K 3$ surfaces with non-symplectic symmetries are algebraic and have been classified in [38] for the $\mathbb{Z}_{2}$ case and $[39,40]$ for the $\mathbb{Z}_{3}$ case. These $K 3$ surfaces can all be used to construct orbifold FSY geometries. The general construction is similar to the constructions given in the previous sections. The main difference being the determination of the torus curvature two-form $\omega$ with the desired transformation property under the $K 3$ discrete group action.

Below we shall show how to go about explicitly writing down the torus twist for algebraic $K 3$ surfaces, and in so doing, construct nonsupersymmetric and singular supersymmetric orbifold geometries. With 
algebraic $K 3$ surfaces, our description becomes effectively purely algebraic. We explain this in the context of a special class of algebraic $K 3$ with a $\mathbb{Z}_{3}$ discrete symmetry group that has only three fixed points and no fixed curves $[39,40] .{ }^{8}$ This unique class of $K 3$ is of particular interest since the singular orbifolds that are constructed from them will only have fixed points and therefore should be easier to resolve. Although we focus on this special class of $K 3$ surfaces, the orbifold construction we give below should be applicable to other algebraic $K 3$ surfaces with a non-symplectic automorphism.

The class of algebraic $K 3$ surfaces that we are interested in can be described, similar to the three-fold branched cover over $\mathbb{P}^{1} \times \mathbb{P}^{1}$, as an intersection of a quadric and a cubic hypersurface in $\mathbb{P}^{4}$. Again, let $\left\{z_{0}, z_{1}, z_{2}\right.$, $\left.z_{3}, z_{4}\right\}$ be the homogeneous coordinates of $\mathbb{P}^{4}$. The class of $K 3$ hypersurface $S$ in $\mathbb{P}^{4}$ is given by the following two equations [39]:

$$
\begin{gathered}
f^{1}=f_{2}\left(z_{0}, z_{1}\right)+b_{1} z_{2} z_{3}+b_{2} z_{2} z_{4}=0, \\
f^{2}=f_{3}\left(z_{0}, z_{1}\right)+b_{3} z_{2}^{3}+g_{3}\left(z_{3}, z_{4}\right)+z_{2} f_{1}\left(z_{0}, z_{1}\right) g_{1}\left(z_{3}, z_{4}\right)=0,
\end{gathered}
$$

where $f_{n}, g_{n}$ are homogeneous polynomials of degree $n$ and $b_{i}$ are non-zero complex constants. Notice that the quadric and cubic equations are invariant under the following discrete action ${ }^{9} \rho$ acting on $S$ :

$$
\rho:\left(z_{0}, z_{1}, z_{2}, z_{3}, z_{4}\right) \rightarrow\left(\zeta^{2} z_{0}, \zeta^{2} z_{1}, \zeta z_{2}, z_{3}, z_{4}\right) .
$$

The solutions of the two hypersurface equations with $\left(z_{0}, z_{1}, z_{2}\right)=(0,0,0)$ give the three fixed points which solve $g_{3}\left(z_{3}, z_{4}\right)=0$.

For instance, we can consider the $K 3$ hypersurface defined by the homogeneous equations

$$
\begin{aligned}
& f^{1}=z_{0}^{2}+z_{1}^{2}+z_{2}\left(z_{3}+z_{4}\right), \\
& f^{2}=z_{1}^{3}+z_{2}^{3}+z_{3}^{3}-z_{4}^{3} .
\end{aligned}
$$

It can be checked that this $K 3$ hypersurface is smooth with $d f^{1} \wedge d f^{2} \neq 0$ on the hypersurface of (6.4). In the coordinate chart $z_{3} \neq 0$, we can use the affine coordinates $Z_{i}=z_{i} / z_{3}$ for $i=0, \ldots, 4$. Then the three fixed points of

\footnotetext{
${ }^{8}$ It follows from the holomorphic Lefschetz formula that any $K 3$ surface that has a $\mathbb{Z}_{3}$ discrete symmetry with no fixed curves must have precisely three fixed points.

${ }^{9}$ To simplify notation, we will in this section use $\rho$ to denote the generator of the discrete symmetry action acting either only on the $K 3$ base, only on the torus fiber, or on the entire six-dimensional geometry $X$. The object of the $\rho$ action should be clear from the context.
} 
(6.3) are located at $\left(Z_{3}, Z_{4}\right)=\left\{(1,1),(1, \zeta),\left(1, \zeta^{2}\right)\right\}$. Similar to (5.10), the holomorphic two form can be written locally for example as

$$
\Omega^{2,0}=\frac{d Z_{0} \wedge d Z_{1}}{-3\left(Z_{2}^{3}+Z_{4}^{2}\right)},
$$

which shows explicitly that the holomorphic two-form transforms nontrivially under the $\mathbb{Z}_{3}$ action as

$$
\rho: \Omega^{2,0} \rightarrow \zeta \Omega^{2,0},
$$

as expected for a non-symplectic automorphism.

To construct orbifold FSY geometries, we need to define the torus curvature twist $\omega=\omega_{1}+\tau \omega_{2}$. The conditions on $\omega,(2.9)$ and (2.10), are that it is an element of $H^{2,0}(S) \oplus H^{1,1}(S)$ with $\omega_{1}, \omega_{2} \in H^{2}(S, \mathbb{Z})$ and that it is primitive.

For the non-supersymmetric orbifold group action that contains a shift on the torus fiber,

$$
\rho:\left(z_{0}, z_{1}, z_{2}, z_{3}, z_{4}, z\right) \rightarrow\left(\zeta^{2} z_{0}, \zeta^{2} z_{1}, \zeta z_{2}, z_{3}, z_{4}, z+1 / 3\right),
$$

the torus curvature $\omega$ must be invariant under $\rho$. Since $\Omega^{2,0}$ is not invariant, we have $\omega_{1}, \omega_{2}$ can only be $(1,1)$ and are elements of the Picard lattice $N=$ $H^{1,1}(S) \cap H^{2}(S, \mathbb{Z})$. In particular, they must be elements of the sublattice $N(\rho) \subset N$ that is invariant under $\rho$. We now show that the $N(\rho)$ is a nonempty lattice and in fact has $\operatorname{rank} \operatorname{rk} N(\rho)=8$. This can be derived using the Lefschetz fixed point formula which relates the Euler characteristic of the fixed point set $S_{\rho}$ with the transformation properties of the de Rham cohomology under $\rho$. We denote the complement lattice $N(\rho)^{\perp}$ of $N(\rho)$ in $H^{2}(S, \mathbb{Z}) . \quad N(\rho)^{\perp}$ is not invariant under $\rho$ and let us assume it has rank $\operatorname{rk} N(\rho)^{\perp}=2 m$. Since the second Betti number $b_{2}=22$, we have $\operatorname{rk} N(\rho)=$ $22-2 m$. The Lefschetz fixed point formula then gives

$$
\begin{aligned}
3=\chi\left(S_{\rho}\right) & =\sum_{k=0}^{4} \operatorname{Tr}\left(\left.\rho\right|_{H^{k}(S, \mathbb{Z})}\right) \\
& =1+\operatorname{rk} N(\rho)+m\left(\zeta+\zeta^{2}\right)+1 \\
& =24-3 m,
\end{aligned}
$$

where we have used the fact that $1+\zeta+\zeta^{2}=0$. This implies that $m=7$ and thus rk $N(\rho)=8$ and $\operatorname{rk} N(\rho)^{\perp}=14$. Thus, we need to choose primitive $\omega_{1}, \omega_{2} \in N(\rho)$ for the torus curvature twist. (The explicit lattice $N(\rho)$ is given in equation (6.11) below.) 
For constructing supersymmetric orbifolds, the orbifold action must act on the torus fiber by a rotation

$$
\begin{aligned}
\theta: & \rightarrow \zeta^{2} \theta \\
\omega & \rightarrow \zeta^{2} \omega
\end{aligned}
$$

so that the holomorphic three-form remains invariant. In order for the torus lattice structure to be compatible with this $Z_{3}$ rotation, we must set the torus complex structure $\tau=\zeta$. The torus curvature two-form $\omega=\omega_{1}+\zeta \omega_{2}$ is now required to have the transformation property $\rho: \omega \rightarrow \zeta^{2} \omega$. Thus, $\omega_{1}, \omega_{2} \in N(\rho)^{\perp}$ and because $\Omega^{2,0}$ transforms as in (6.6), we must again have $\omega \in H^{1,1}(S)$. Note that elements in $N(\rho)^{\perp}$ are real and generally, in addition to $(1,1)$ components, also have $(2,0)$ and $(0,2)$ components. Thus to ensure that $\omega$ is purely $(1,1)$, we need to determine explicitly the complex structure of $S$. To do so will require some knowledge of the lattice $L$ of the second integral cohomology, $H^{2}(S, \mathbb{Z})$, which we now explain. (For more details on $L$, see for example [41].)

$L$ is a self-dual unimodular lattice. For elements $x_{i} \in L$, there is a natural bilinear form on $L$ given by the integral

$$
d_{i j}=\left(x_{i}, x_{j}\right)=\int_{S} x_{i} \wedge x_{j} .
$$

The bilinear form on $L$ is that of the lattice $U^{3} \oplus\left(E_{8}\right) \oplus\left(E_{8}\right)$. Here, $U$ denotes the hyperbolic lattice defined by $\left(\begin{array}{ll}0 & 1 \\ 1 & 0\end{array}\right)$ and $A_{n}, D_{n}, E_{n}$ denote the "negative" definite lattice of the corresponding Lie algebra root system. The form is thus given by the negative of the Cartan matrix.

Of course, $N(\rho)$ and $N(\rho)^{\perp}$ are both sublattices of $L$. For $K 3$ surfaces with a $\mathbb{Z}_{3}$ action having only fixed points, they take the form $[39,40]$

$$
N(\rho)=U(3) \oplus E_{6}^{*}(3), \quad N(\rho)^{\perp}=A_{2}(-1) \oplus A_{2}^{6},
$$

where for example $U(3)$ denotes the lattice with three times the bilinear form of $U$ and $E_{6}^{*}$ denotes the lattice dual to the $E_{6}$ lattice. Each sublattice in $N(\rho)^{\perp}$ contains an order three discrete symmetry action $\rho$. For the lattice $A_{2}$ associated with the negative Cartan matrix $\left(\begin{array}{cc}-2 & 1 \\ 1 & -2\end{array}\right)$, let $\{e, f\}=\left\{\left(\begin{array}{l}1 \\ 0\end{array}\right),\left(\begin{array}{l}0 \\ 1\end{array}\right)\right\}$ be the basis vectors that have the inner product $(e, e)=(f, f)=-2$ and $(e, f)=1$. The $\mathbb{Z}_{3}$ symmetry action on the lattice 
can be described by

$$
\begin{aligned}
e & \rightarrow f \\
f & \rightarrow-e-f .
\end{aligned}
$$

Under this action, the linear combinations $(e-\zeta f)$ and $(f-\zeta e)$ transform as

$$
\rho: \begin{aligned}
& e-\zeta f \rightarrow \zeta(e-\zeta f) \\
& f-\zeta e \rightarrow \zeta^{2}(f-\zeta e)
\end{aligned}
$$

and span an eigenbasis of two forms with eigenvalues $\zeta$ and $\zeta^{2}$, respectively.

We can now explicitly write down the complex structure $S$, or equivalently, the associated holomorphic two form. By $(6.6), \Omega^{2,0} \in N(\rho)^{\perp} \otimes \mathbb{C}$. We can express $\Omega^{2,0}$ as a linear combination of the basis elements of the lattice $N(\rho)^{\perp}=A_{2}(-1) \oplus A_{2}^{6}$

$$
\Omega^{2,0}=B_{0}\left(e_{0}-\zeta f_{0}\right)+\sum_{i=1}^{6} B_{i}\left(e_{i}-\zeta f_{i}\right),
$$

where $B_{0}$ and $B_{i}$ for $i=1, \ldots, 6$ are complex constants and $\left\{e_{0}, f_{0}\right\},\left\{e_{i}, f_{i}\right\}$ are, respectively, the basis elements of $A_{2}(-1)$ and the six $A_{2}$ 's. We shall take all pairs of $\{e, f\}$ to transform under $\rho$ as in (6.12). The constants $B_{0}, B_{i}$ determine the complex structure and equivalently the periods with respect to a specified marking of two cycles on the $K 3$. They are constrained by three consistency conditions. The two standard ones are

$$
\begin{gathered}
\int_{S} \Omega^{2,0} \wedge \Omega^{2,0}=0 \\
\int_{S} \Omega^{2,0} \wedge \bar{\Omega}^{2,0}=3\left(\left|B_{0}\right|^{2}-\sum_{i=1}^{6}\left|B_{i}\right|^{2}\right)>0 .
\end{gathered}
$$

The first condition is automatically satisfied by an $\Omega^{2,0}$ expressed as in (6.14). The second by itself is a weak condition and can be easily satisfied.

The third consistency condition involves the Picard lattice $N$. In general, the Picard lattice $N$ consists of the invariant $N(\rho)$ and also any elements in $N(\rho)^{\perp}$ that are $(1,1)$, i.e., $N(\rho)^{\perp} \cap \Omega^{\perp}$. Let $T=N^{\perp}$ be the complement lattice to $N$. For a generically chosen complex structure $\Omega^{2,0}$, we will have $T=N(\rho)^{\perp}$ and $N=N(\rho)$, that is all elements in $N(\rho)^{\perp}$ will have a $(2,0)$ and a $(0,2)$ part. But for special complex structures, only $T \subset N(\rho)^{\perp}$ and $N(\rho) \subset N$. Nevertheless, for elements in the Picard lattice that transforms non-trivially under $\rho$, it is necessary that there exists no element $h$ with $(h, h)=-2$. For if such exists, then by the Riemann-Roch theorem applied 
to $K 3$ surfaces, there is an effective divisor $\pm h$ which under the action $\rho$ : $\pm h \rightarrow \pm \zeta h$ or $\pm \zeta^{2} h$ which is not possible [42]. Thus, for instance, choosing $B_{0}=1$ and $B_{i}=0$ would lead to $(-2)$-curves for $h=e_{i}$ or $f_{i}$ and would not be valid. A consistent choice would be

$$
B_{0}=3, B_{1}=1, \quad \text { and } \quad 0<B_{i} \leq 1 \quad \text { for } i=2, \ldots, 6
$$

for $B_{i}$ sufficiently generic.

Having established the lattice structure and the complex structure, we can now determine the possible torus curvature two-form $\omega$. As mentioned, under the discrete symmetry action, $\omega$ must transform as in (6.9). Matching the transformation property of $\omega$ with those in (6.13) implies that $\omega$ can be written as a linear combination

$$
\omega=C_{0}\left(f_{0}-\zeta e_{0}\right)+\sum_{i=0}^{6} C_{i}\left(f_{i}-\zeta e_{i}\right),
$$

where $C_{0}$ and $C_{i}$ 's are complex constants. Since we require that $\omega$ does not have an $(0,2)$ component, we have the additional constraint

$$
\int_{S} \Omega^{2,0} \wedge \omega=-3 \zeta\left(B_{0} C_{0}-\sum_{i=0}^{6} B_{i} C_{i}\right)=0 .
$$

Note that $\int_{S} \bar{\Omega}^{0,2} \wedge \omega=0$ is automatically satisfied for $\omega$ given by (6.18).

For the choice of complex structure specified by (6.14) and (6.17), we can for example choose $C_{0}=1, C_{1}=3$, and all other $C_{i}=0$ which gives

$$
\omega=\omega_{1}+\zeta \omega_{2}=\left(f_{0}+3 f_{1}\right)+\zeta\left(-e_{0}-3 e_{1}\right) \in H^{1,1}(S) .
$$

We see that although $\omega_{1}, \omega_{2} \in H^{2}(S, \mathbb{Z})$ only, the combination $\omega=\omega_{1}+$ $\zeta \omega_{2} \in H^{1,1}(S)$ as required. Furthermore, for $\omega$ in (6.20) we also have $\int_{S} \omega \wedge$ $\bar{\omega}=-24$ (having set $A=\tau_{2}$ in (2.11)) which would give us a $\mathcal{N}=2$ FSY geometry with trivial gauge bundle.

Orbifolding the FSY geometry by the $\mathbb{Z}_{3}$ action,

$$
\rho:\left(z_{0}, z_{1}, z_{2}, z_{3}, z_{4}, z\right) \rightarrow\left(\zeta^{2} z_{0}, \zeta^{2} z_{1}, \zeta z_{2}, z_{3}, z_{4}, \zeta^{2} z\right) .
$$

The resulting supersymmetric orbifold geometry has $3 \times 3=9$ fixed points. (The $\mathbb{Z}_{3}$ action on the torus, $\rho: \theta \rightarrow \zeta^{2} \theta$, also lead to three fixed points.) Each fixed point is locally a $\mathbb{C}^{3} / \mathbb{Z}_{3}$ orbifold that can be minimally resolved by a $\mathbb{P}^{2}$. Thus the resolved manifold of this singular orbifold has Euler characteristic $\chi(\widehat{Y})=(0-9) / 3+9 \chi\left(\mathbb{P}^{2}\right)=24$. For this orbifold with only 
nine fixed points, there is already a candidate local model that we can use to resolve each singularity of the geometry. This is the well-known local Calabi-Yau metric that resolves $\mathbb{C}^{3} / \mathbb{Z}_{3}[22,43]$. However, careful analysis is required to verify that such gluing-in can preserve the required supersymmetry and anomaly conditions.

\section{Discussions}

We have constructed new non-Kähler heterotic geometries $Y=X / \Gamma$ by orbifolding the torus bundle over $K 3$ base geometry by a discrete symmetry group $\Gamma$. The orbifolds $Y$ are clearly topologically distinct from the starting torus bundle geometry $X$. For smooth orbifold models, the first fundamental group $\pi_{1}(Y)$ additionally contains the symmetry group $\Gamma$. For singular orbifolds where $G$ has a fixed locus set, the resolution of singularities will generically give a non-zero Euler characteristic for the resolved manifold $\widehat{Y}$.

The orbifold models we have constructed can be supersymmetric or break all supersymmetries. We have given explicit constructions of orbifold solutions where the $K 3$ base of the FSY model is either a Kummer $K 3$ or has a branched covering description or a more general algebraic description. Those orbifold geometries arising from a shift action on the fiber can still be viewed as having a fiber space structure. The base is now the $K 3$ orbifolded by the discrete action and the complex structure of the torus jumps along the fixed locus set of the discrete action. Rotation actions on the torus fiber may result in orbifold models with singularities, which potentially can have a non-vanishing Euler characteristic as well as a non-vanishing number of generations. The smooth resolution of these non-Kähler singular orbifold solutions is an important question that shall be addressed in future work.

The smooth non-supersymmetric geometries we have constructed are each an elliptic fibration over a complex surface. For example, the one in Section 5.1 utilizing a branched covered $K 3$ surface is an elliptic fibration over a $\mathbb{P}^{1} \times \mathbb{P}^{1}$ base. Being non-supersymmetric, the holomorphic three form of the FSY geometry has been projected out. It is thus interesting to ask whether these smooth torsional elliptically fibered three-folds more generally can support a no-where vanishing holomorphic three-form. Such would lead directly to smooth supersymmetric heterotic geometries. Some preliminary metric ansätze for these elliptically fibered three-folds has been proposed in [44]. Interestingly, from the perspective of F-theory and heterotic string duality $[18,45]$, elliptically fibered three-folds should be dual to F- or M-theory flux compactifications on Calabi-Yau four-folds [46] that are $K 3$ fibrations. Recent work using this approach appears to indicate the existence of such class of heterotic flux compactifications [47]. 
It is worthwhile to emphasize that the FSY geometries, at least for those that preserve $\mathcal{N}=2$ SUSY, have a conformal field theory description [17] and represent a class of string vacua valid to all orders in $\alpha^{\prime}$. The construction of geometric orbifold models described here should therefore have an analogous orbifold description from the CFT perspective. The CFT orbifold models that are constructed from freely acting discrete groups should just consist of a projection. For the non-freely acting ones, the resolution of singularities should correspond to the addition of twisted sector modes. It would be interesting to work out the CFT description of the orbifold models constructed herein.

An important question that we leave for future work is whether the nonsuper-symmetric orbifolds are stable in the $g_{s}$ loop expansion. Some beautiful examples of non-supersymmetric stable orbifolds were constructed in [48]. Non-supersymmetric unstable orbifolds can lead to an interesting decay process as has been discussed in the recent literature $[49,50]$. In particular in [50] non-compact non-supersymmetric orbifolds were shown to decay into supersymmetric ALE spaces. It would be interesting to see if an analysis along the lines of [49] can be performed for the heterotic orbifold models we have constructed.

\section{Acknowledgments}

We thank A. Adams, A. Bergman, K. Becker, R. Donagi, J.-X. Fu, S. Kachru, B. Lian, J. Lapan, D. Morrison, K. Oguiso, A. Sarti, A. Strominger, A. Subotic, A. Todorov, and C. Vafa for useful discussions and correspondence. M.B. would like to thank the Harvard Department of Physics for hospitality where part of this work was carried out. M.B. and L.-S.T. would also like to thank the hospitality of the 6th-Simons Workshop in Mathematics and Physics at SUNY Stony Brook near the completion of this work. M.B. is supported by NSF grant PHY-0505757 and the University of Texas A\&M. L.-S. Tseng is supported in part by NSF grant PHY-0714648. S.-T.Y. is supported in part by NSF grants DMS-0306600 and PHY-0714648.

\section{References}

[1] V. Bouchard and R. Donagi, An SU(5) heterotic standard model, Phys. Lett. B633 (2006), 783, arXiv:hep-th/0512149.

[2] V. Braun, Y. H. He, B. A. Ovrut and T. Pantev, A heterotic standard model, Phys. Lett. B618 (2005), 252, arXiv:hep-th/0501070. 
[3] B. Andreas and G. Curio, Heterotic models without fivebranes, J. Geom. Phys. 57 (2007), 2136, arXiv:hep-th/0611309;

Deformations of bundles and the standard model, Phys. Lett. B655 (2007), 290, arXiv:0706.1158;

Extension bundles and the standard model, JHEP 0707 (2007), 053, arXiv: hep-th/0703210.

[4] G. B. Cleaver, A. E. Faraggi and D. V. Nanopoulos, String derived MSSM and M-theory unification, Phys. Lett. B455 (1999), 135, arXiv:hep-ph/9811427.

[5] R. Donagi and M. Wijnholt, Model building with F-theory, arXiv:0802.2969.

[6] C. Beasley, J. J. Heckman and C. Vafa, GUTs and exceptional branes in F-theory - I, arXiv:0802.3391.

[7] A. Strominger, Superstrings with torsion, Nucl. Phys. B274 (1986), 253.

[8] C. M. Hull, Superstring compactifications with torsion and space-time supersymmetry, in 1st Torino Meeting on Superunification and Extra Dimensions, September 1985, Torino, Italy, eds. R. D'Auria and D. Fre, World Scientific, Singapore, 1986, 347;

Anomalies, ambiguities and superstrings, Phys. Lett. B167 (1986), 51; Compactifications of the heterotic superstring, Phys. Lett. B178 (1986), 357.

[9] B. de Wit, D. J. Smit and N. D. Hari Dass, Residual supersymmetry of compactified $d=10$ supergravity, Nucl. Phys. B283 (1987), 165.

[10] J.-X. Fu and S.-T. Yau, The theory of superstring with flux on nonKähler manifolds and the complex Monge-Ampére equation, J. Differential Geom. 78 (2008), 369, arXiv:hep-th/0604063.

[11] K. Becker, M. Becker, J.-X. Fu, L.-S. Tseng and S.-T. Yau, Anomaly cancellation and smooth non-Kähler solutions in heterotic string theory, Nucl. Phys. B751 (2006), 108, arXiv:hep-th/0604137.

[12] E. Goldstein and S. Prokushkin, Geometric model for complex nonKähler manifolds with SU(3) structure, Commun. Math. Phys. 251 (2004), 65, arXiv: hep-th/0212307.

[13] K. Becker, M. Becker, K. Dasgupta and P. S. Green, Compactifications of heterotic theory on non-Kähler complex manifolds I, JHEP 0304 (2003), 007, arXiv:hep-th/0301161.

[14] E. Calabi and B. Eckmann, A class of compact, complex manifolds which are not algebraic, Ann. Math. 58 (1953), 494.

[15] K. Dasgupta, G. Rajesh and S. Sethi, $M$ theory, orientifolds and $G$-flux, JHEP 9908 (1999), 023, arXiv:hep-th/9908088. 
[16] K. Becker and K. Dasgupta, Heterotic strings with torsion, JHEP 0211 (2002), 006, arXiv:hep-th/0209077.

[17] A. Adams, M. Ernebjerg and J. M. Lapan, Linear models for flux vacua, Adv. Theor. Math. Phys. 12 (2008), 821, arXiv:hep-th/0611084.

[18] K. Becker, M. Becker, P. S. Green, K. Dasgupta and E. Sharpe, Compactifications of heterotic strings on non-Kähler complex manifolds II, Nucl. Phys. B678 (2004), 19, arXiv:hep-th/0310058.

[19] P. Candelas, G. T. Horowitz, A. Strominger and E. Witten, Vacuum configurations for superstrings, Nucl. Phys. B258 (1985), 46.

[20] L. J. Dixon, J. A. Harvey, C. Vafa and E. Witten, Strings on orbifolds, Nucl. Phys. B261 (1985), 678;

L. J. Dixon, J. A. Harvey, C. Vafa and E. Witten, Strings on orbifolds 2, Nucl. Phys. B274 (1986), 285.

[21] S.-T. Yau, Compact three dimensional Kähler manifolds with zero Ricci curvature, in Symposium on Anomalies, Geometry, and Topology, eds. W. A. Bardeen and A. R. White, 1985, 395.

[22] A. Strominger and E. Witten, New manifolds for superstring compactification, Commun. Math. Phys. 101 (1985), 341.

[23] T. Hübsch, Calabi-Yau manifolds: a bestiary for physicists, World Scientific, New Jersey, 1992.

[24] P. S. Aspinwall, K3 surfaces and string duality, hep-th/9611137.

[25] B. R. Greene, String theory on Calabi-Yau manifolds, arXiv:hep-th/9702155.

[26] G. L. Cardoso, G. Curio, G. Dall'Agata, D. Lust, P. Manousselis and G. Zoupanos, Non-Kähler string backgrounds and their five torsion classes, Nucl. Phys. B652 (2003), 5, arXiv:hep-th/0211118.

[27] J. P. Gauntlett, D. Martelli and D. Waldram, Superstrings with intrinsic torsion, Phys. Rev. D69 (2004), 086002, arXiv:hep-th/0302158.

[28] M. Becker, L.-S. Tseng and S.-T. Yau, Heterotic Kähler/nonKähler transitions, Adv. Theor. Math. Phys. 12 (2008), 1151, arXiv:0706.4290.

[29] M. Cyrier and J. M. Lapan, Towards the massless spectrum of nonKaehler heterotic compactifications, Adv. Theor. Math. Phys. 10 (2007), 853, arXiv:hep-th/0605131.

[30] T. Kimura and P. Yi, Comments on heterotic flux compactifications, JHEP 0607 (2006), 030, arXiv: hep-th/0605247.

[31] J.-X. Fu, L.-S. Tseng and S.-T. Yau, Local heterotic torsional models, arXiv:0806. 2392.

[32] N. Hitchin, Compact four-dimensional Einstein manifolds, J. Diff. Geom. 9 (1974), 435. 
[33] C. Vafa and E. Witten, Dual string pairs with $N=1$ and $N=2$ supersymmetry in four dimensions, Nucl. Phys. Proc. Suppl. 46 (1996), 225, arXiv:hep-th/9507050.

[34] J. A. Harvey, D. A. Lowe and A. Strominger, $N=1$ string duality, Phys. Lett. B362 (1995), 65, arXiv:hep-th/9507168.

[35] D. G. Markushevich, M. A. Olshanetsky and A. M. Perelomov, Description of a class of superstring compactifications related to semi-simple Lie algebras, Commun. Math. Phys. 111 (1987), 247.

[36] S.-S. Roan and S.-T. Yau, On Ricci flat 3-fold, Acta Math. Sinica 3(3) (1987), 256.

[37] M. B. Green, J. H. Schwarz and E. Witten, Superstring theory, Vol. 2, Cambridge University Press, 1987.

[38] V. V. Nikulin, Factor groups of groups of automorphisms of hyperbolic forms with respect to subgroups generated by 2-reflections, J. Soviet Math. 22 (1983), 1401.

[39] M. Artebani and A. Sarti, Non-symplectic automorphisms of order 3 on K3 surfaces, arXiv:0801.3101.

[40] S. Taki, Classification of non-symplectic automorphisms or order 3 on K3 surfaces, arXiv:0802:1956.

[41] D. R. Morrison, On K3 surfaces with large Picard number, Invent. Math. 75 (1984), 105.

[42] V. Alexeev and V. V. Nikulin, Del Pezzo and K3 surfaces, Mathematical Society of Japan, Tokyo, 2006.

[43] E. Calabi, Métriques Kählériennes et fibrés holomorphes, Ann. Sci. Ec. Norm. Super. 12 (1979), 269.

[44] M. Becker, L.-S. Tseng and S.-T. Yau, unpublished.

[45] D. Morrison and C. Vafa, Compactifications of F-theory on Calabi-Yau threefolds-II, arXiv:hep-th/9603161.

[46] K. Becker and M. Becker, M-theory on eight-manifolds, Nucl. Phys. B477 (1996), 155, arXiv:hep-th/9605053.

[47] Talk of K. Becker at String phenomenlogy 2008, May 28-June 1, 2008, University of Pennsylvania;

K. Becker and S. Sethi, Torsional heterotic geometries, Nucl. Phys. B820, 2009, 1, arXiv:0903.3769.

[48] S. Kachru and E. Silverstein, 4d conformal theories and strings on orbifolds, Phys. Rev. Lett. 80 (1998), 4855, arXiv: hep-th/9802183.

[49] A. Adams, J. Polchinski and E. Silverstein, Don't panic! Closed string tachyons in ALE space-times, JHEP 0110 (2001), 029, arXiv: hep-th/0108075.

[50] G. T. Horowitz, J. Orgera and J. Polchinski, Nonperturbative instability of $A d S_{5} \times S^{5} / Z_{k}$, Phys. Rev. D77 (2008), 024004, arXiv:0709.4262. 
